\title{
Effects of Perinatal Protein-Nacl Diets on Offspring's Blood Pressure and Renal Function in Lewis Rats
}

\author{
Jong Y. Lee* and Silvia H. Azar
}

Department of Medicine, University of Minnesota School of Medicine, Minneapolis, Minnesota, USA

\begin{abstract}
Perinatal diets may affect the cardiovascular-renal functions of offspring. To understand effects of maternal diet on the renal function and blood pressure (BP) of offspring, protein (10\% low, LP; $23 \%$ normal, NP) and/or $\mathrm{NaCl}$ ( $4 \%$ high salt, HS; $0.6 \%$ normal, NS) diets were started at pre-pregnancy through pups' weaning to either a $4 \%$ high $\mathrm{NaCl}(\mathrm{hs})$ or $0.6 \% \mathrm{NaCl}(\mathrm{ns})$ diet. Telemetered BP data was analyzed by methods of linear least square rhythmometry. Systolic BPs (circadian mean \pm SE mm Hg) were: NPNSns, 131 \pm 2 ; NPNShs, 137 \pm 2 ; NPHSns, 137 \pm 0.2 ; NPHShs, 134 \pm 3 ; LPNSns, $138 \pm 1$; LPNShs, 138 \pm 0.6 ; LPHSns, 135 \pm 2 ; LPHShs, 142 \pm 2 . Offspring in NPNShs and NPHSns had significantly increased SBPs versus NPNSns (both $\mathrm{P}<0.05)$. Most LP-offspring had increased SBP $(\mathrm{P}<0.01$ to $<0.05)$ and lower body weight (BW) with smaller glomerular filtration rate changes (renal reserve, RR-GFR) following overnight acute highprotein loads: RR-GFRs (inulin, $\mathrm{ml} / \mathrm{min} / \mathrm{g}$ kidney) for groups stated above were, respectively: $0.935 ; 0.927 ; 0.537 ;-0.064$; $-0.229 ; 0.057 ;-0.515 ;-0.404$. The kidney weight/BW ratio of offspring was higher on hs- than on ns-diets (all $\mathrm{P}<0.001)$. Rats on a low caloric diet had reduced sclerotic glomerular numbers compared to those on normal diets (11.2 \pm 1 vs. $15.7 \pm 2, \mathrm{P}<0.001$ ), though glomerular numbers were similar in both groups.

In summary, perinatal LP-HS diets significantly affected the BW, BP, renal injuries and kidney function of offspring. RR was seriously reduced, especially among offspring in hs- and perinatal LP groups. The most interesting result was the glomerular maturation staging in the pups, which suggests delayed nephrogenesis by a maternal LP diet.
\end{abstract}

Keywords: Circadian blood pressure, Glomerular filtration rate, perinatal protein-salt diets, renal injury, renal maturation, renal reserve.

\section{INTRODUCTION}

Genetic and environmental factors play an important role in disease expression on offspring by exerting diverse and predominant modulatory effects [1-3]. Cardiac disease and chronic kidney disease (CKD) commonly coexist, sharing cardiovascular disease (CVD) risk factors such as hypertension, dyslipidemia and diabetes. CKD is an independent predictor of MI, stroke, and mortality risk among men and women younger than 55 and 65 years of age, respectively, which has been defined by levels of estimated glomerular filtration rate (eGFR) as markers of kidney damage [4-6].

Perinatal dietary imbalance may induce structural changes in a progeny's body that can lead to injury, especially given genetic susceptibility $[1,3]$. Some authors have addressed epigenetic effects - in the relationship between longevity and food availability in the transgeneration response to swings in nutrition - on the regulation of reproduction with disease risk [7]. The effects of perinatal nutrition impact health and disease later in adult life in manifesting the altered epigenetic regulation of gene expression caused by defective epigenetic regulation [8]. Barker et al. hypothesized that adult disease may originate from fetal stage developmental plasticity and compensatory biological phenomena [9].

*Address correspondence to this author at the P.O. BOX 14945, Minneapolis, MN 55414, USA; Tel \& Fax: (612) 379-2467;

E-mail: leexx154@umn.edu
With respect to epigenetic effects, perinatal dietary salt and protein may be involved in the development of the kidney and programming blood pressure (BP) during morphogenesis, organogenesis and glomerulogenesis, which may affect an offspring's renal reserve (RR) and BP. The altered maturation patterns of glomerular and/or tubular mass can trigger adaptive mechanisms that lead to glomerular hypertension later in life [10]. Maternal stress and nutritional conditions modulated BP and renal injuries at all stages, altered glomerular filtration and resulted in glomerulosclerosis $[2,3$, $11,12]$. Excessive salt intake can be hypertensinogenic in susceptible individuals, adversely affecting target organs especially among the aging population, as renal function declines [11]. In certain Japanese populations, cerebrovascular complications, which are associated with high salt/low protein diets [13]. The effect of dietary salt on hypertension [13] and maternal protein deficiency on behavioral parameters in children [14-16] suggest that a great segment of the population may consume food with high salt/low protein content.

Protein deficiency during perinatal stages induces reduced plasma volume, cardiac output and uteroplacental blood flow [17], and decreased vascular growth with high capillary luminal diameters in the neocortex among suckling dams [18], suggesting a vasodilated vascular mass to respond to hypertension and hypoxia to form collateral vessels after sudden vascular occlusion [19]. The kidneys of suckling offspring with maternal protein deficiency showed altered 
glomerular maturation and resulted in a reduced glomerular number as adults [10, 20]. Meanwhile other studies have reported that the glomerular injury index and urine protein excretion were ameliorated in a low calorie, unrestricted protein diet in uninephrectomized spontaneously hypertensive rats (SHR) [12]. Heterogeneous glomerular growth in susceptible genetic strains needs high pressure gradients and/or glomerular hypertension to adapt and maintain glomerular filtration $[21,22]$. These adaptations, which eventually lead to glomerulosclerosis $[15,22]$, can be among the factors that account for differences in renal damage induced by hypertension and age in two genetic models of hypertension [14, $22,23]$. In renal function tests, some studies suggest that amino acid (AA) infusion induces vasodilation and increases the GFR in normotensive rats, but not in SHR [24]. As shown, a perinatal-environmental experience in fetal programming exerts a long-term influence on cardiovascular functions, leading to disease in adulthood.

Under the hypothesis, we examined perinatal dietary protein and salt effects on the kidney maturation of offspring with a moderate protein restriction and subtoxic levels of dietary salt and assessed progeny responses to the perinatal and post-maternal diet on body weight (BW), SBP, GFR and $\mathrm{RR}$ in the Lewis rat, which is known to have a high degree of homozygosity [25].

\section{MATERIALS AND METHODS}

\section{Animals}

Ten-week old Lewis female rats and 14-15 week old vasectomized and proven breeder males were purchased from Charles River (Wilmington, MA) and were housed at the University animal facilities (a light-cycle from 04:00 to 18:00) with standard rat chow (Purina Rodent Chow, St. Louis, MO) and freely accessible drinking water. After 7 days of adaptation, female rats were stratified according to $\mathrm{BW}( \pm 10 \mathrm{~g})$ and $\mathrm{BP}( \pm 10 \mathrm{~mm} \mathrm{Hg})$, and randomly assigned to ensure balanced conditions for all study groups. To minimize circadian variability, manual $\mathrm{BP}$ and $\mathrm{BW}$ were obtained between 14:00 and 18:00h. Pregnancy was maintained starting at the age of 12-13 weeks. Dams were kept on the designated special diet throughout pregnancy starting at 21-27 days prior to mating to the end of lactation [1]. To obtain a sufficient number of pups within 4 hours of delivery, 12 dams (each 4 maternal group $\times 3$ ) were mated to assign at least 5-7 pups into the high (hs) and normal salt (ns) groups from each maternal group at the end of the weaning period. For glomerular maturation and proteinuria studies, as well as the restricted calorie diet studies, another two dams in each of the four maternal diet groups, and two additional normal diet dams were added. After weaning, dams were sacrificed using barbiturate overdose in accordance with the recommendations of the Panel on Euthanasia of the American Veterinary Medical Association.

Offspring's age for the study varied from Day-0 to 15 weeks. At weaning, pups were randomly assigned to each designated group according to similar birth times and BWs to ensure group balance, and started on the designated diets ( $4 \%$ high salt, hs or $0.6 \%$ normal salt, ns, total 8 groups) with freely accessible water under a light-cycle from 06:00 to 18:00. All diets were balanced nutrition with isocaloric, similar $\mathrm{K}$, a $\mathrm{Ca} / \mathrm{P}$ of 1.25 and a sugar/starch ratio of 1.00 , pelleted and refrigerated to maintain freshness before using.

The study was approved by the institutional animal care and use committee, and all of the experimental procedures were carried out in accordance with the relevant guidelines (i.e., the Guide for the Care and Use of Laboratory Animals) published by the United States National Institutes of Health and the University of Minnesota Animal Use guidelines.

\section{Study Design}

A diagrammatic overall study design is illustrated in Fig. (1) for a quick review of the study protocol. To test maternal protein and salt influences on the SBP and renal injury susceptibility of offspring, the current study was designed for pre-pregnancy and pregnancy in protein-salt diets imposed according to the study design, with 2 levels of salt $(0.6 \%$ normal, NS and 4\% high, HS) and/or 2 levels of protein (10\% low, LP and 23\% normal, NP) diets, which went from pre-pregnancy through pups' weaning stage, to either a $4 \%$ high $\mathrm{NaCl}$ (hs) or $0.6 \%$ normal $\mathrm{NaCl}$ (ns) diet. The maternal diet groups were: 1) NPNS = normal protein/normal salt; 2) NPHS = normal protein/high salt; 3) LPNS = low protein/normal salt; and 4) LPHS: low protein/ high salt. Baseline food intake (FI), SBP, urine volume (UV) and GFR were measured and RR-FI, RR-SBP, RR-UV and RR-GFR were assessed during the morning hours (7:00 AM-11:00 AM) after maximal food intake overnight (18:00-04:00), followed the next night by high protein (hp, 43\%) loads. RR (post-hp loaded value - basal value) was computed in rats (basal - hp food intake) within \pm 2 grams (each group had at least 5 rats). All other dietary components were similar in all study groups. For this study, special diets were made by Tekled Premier (Madison, WI), based on the American Institute of Nutrition Rodent Diets [3, 22].

\section{PROCEDURES}

\section{Female Synchronization and Offspring Procedures}

Lewis female rats were tested for behavioral estrous at the 20:00 hour with vasectomized sterile-proven males, as in our previous studies [1]. Then, the positive females were randomly assigned to and started on one of the 4 protein-salt dietary combinations, and maintained on those diets from 21 27 days prior to mating through pregnancy and lactation (4 maternal groups). Following the pre-pregnancy period, the dams were re-tested for estrous cycle receptive days for the next 6 days (rat's cycle: 4-5 days) and positive rats were mated overnight with the same strain of fertile males. Males were on a corresponding special diet only on mating nights. At birth, dams and pups were weighed and, the nursing dam litter size was normalized to 8 pups. The same diet was continued until the pups' weaning at 21 days. Food was placed at a height accessible only to mothers to ensure that pups received only maternal milk. Dams were sacrificed after the pups' weaning. Due to variations in fetal programming and of the female estrous cycle $[26,27]$, as well as a difference in prenatal sex malnutrition, especially in endothelial nitric oxide synthase activities [28], male pups were only used in the current study to minimize the sex variability. Pups were randomly weaned to either a normal- or a high-salt diet, according to the study design (8 study groups). The offspring's 


\section{Study Design:}

\begin{tabular}{|c|c|}
\hline \multicolumn{2}{|c|}{$\begin{array}{c}\text { Stage Protocole and Diet } \\
\text { Perinatal Stage }\end{array}$} \\
\hline 1 week: & $\begin{array}{l}\text { Purchase } 10 \text { week-old female, } \\
\text { \&14-15 week-old male, } \\
\text { regular Purina chow, tap water. }\end{array}$ \\
\hline 21-27 days: & $\begin{array}{l}\text { Female Prepregnancy, base WT, BP } \\
10 \% \text { protein (LP)/23\% protein (NP) diets } \\
\text { and } 0.6 \% \text { (NS) } / 4 \%(\mathrm{HS}) \mathrm{NaCl} \text { diets, } \\
\text { tap water. Total } 4 \text { groups. }\end{array}$ \\
\hline & $\begin{array}{l}\text { Pregnancy, WT, BP, the same diet as } \\
\text { the above. Total } 4 \text { groups. }\end{array}$ \\
\hline \multicolumn{2}{|c|}{ Male Offspring } \\
\hline Day-0: \& 7: & $\begin{array}{l}4 \text { groups: NPNS, NPHS, LPNS, LPHS, } \\
\text { maternal milk only, WT; glomerular } \\
\text { maturation examined in offspring } \\
\text { subgroups from } 4 \text { maternal groups. }\end{array}$ \\
\hline Day-19/21: & $\begin{array}{l}\text { - W T, weaned at day-21 into } 0.6 \% \text { (ns) } \\
\text { or } 4 \% \text { (hs) } \mathrm{NaCl} \text { diet, tap water. } \\
\text { Total } 8 \text { groups. }\end{array}$ \\
\hline Day-21: & $\begin{array}{l}\text { - Calorie restriction for } 4 \text { weeks: glomerular } \\
\text { injury comparison in ns-offspring subgroups } \\
\text { from } 4 \text { maternal groups, }\end{array}$ \\
\hline Day-56: & \begin{tabular}{c|c} 
24-h urine protein level in ns-offspring \\
subgroups from 4 maternal groups.
\end{tabular} \\
\hline Week14-15: & \begin{tabular}{l|l}
- & SBP monitoring, $24-\mathrm{h}$ food intake count, \\
24-h urine collection, BW, GFR, KW from \\
8 study groups, ns/hs-offspring diets.
\end{tabular} \\
\hline
\end{tabular}

Fig. (1). Diagram of Study Design.

diet was a standard rodent diet except the salt content with a $4 \%$ high (hs) or $0.6 \%$ normal $\mathrm{NaCl}$ (ns).

\section{Glomerular Maturation Study}

In separate groups, pups' kidneys were examined at birth (day-0) and day-7 to compare their glomerular maturation levels in each maternal diet group. Under methohexital anesthesia, the kidneys of each rat were perfusion fixed at day- 0 and day-7 with 5\% neutral formalin through the abdominal aorta for $10 \mathrm{~min}$ under a perfusion pressure of $80 \mathrm{~mm} \mathrm{Hg}$. Then, the kidneys were removed and immersed in $10 \%$ neutral formalin to complete the fixation. Embedded tissue blocks were sectioned at $15 \mu \mathrm{m}$ thick and all cross sections were assessed from the cortex and juxtamedullary areas. We graded glomerular maturation (GM) as follows: Stage 0: metanephric mesenchyma; Stage I: S body vesicle; Stage II: spherical cup shape, capsular epithelium, 1-2 capillaries, Bowman space not well defined; Stage III: 6-8 capillaries or more, well-defined Bowman space. For the microscopic readings, the multiple serial histological sections of both kidneys in each rat were made and examined under the graded microscope. Each pair of sections was mounted on two light microscopes with modified projection and corresponding fields on the two sections and the total renal cross sectional area was determined. The examiner was blinded on the study samples. For the proteinuria measurement, 24-h urine was collected in individual metabolic cages and protein excretion was measured by spectrophotometer frequently used in our laboratories [10, 22-24, 29].

\section{Effect of Calorie Restriction on Glomerular Injury}

In a subset of pups from the normal maternal diet group, we also examined glomerular numbers and sclerotic injury scores in a $1 / 3$ reduced calorie diet (LC) with freely accessible drinking water for 4 weeks without protein restriction and compared them with pups on a diet without calorie reduction (NC) (average $\mathrm{LC}=10$ versus $\mathrm{NC}=15$ grams/day, 5 rats in each group). Under pentobarbital anesthesia, the kidneys of each rat were fixed by perfusion with $5 \%$ neutral formalin through the abdominal aorta for 15 min under a perfusion pressure of $110 \mathrm{~mm} \mathrm{Hg}$, and the kidneys were removed for immersion in $10 \%$ neutral formalin to complete the fixation. Embedded tissue blocks were sectioned at 15 $\mu \mathrm{m}$ and all glomeruli/cross section were assessed from the cortex and juxtamedullary areas. The glomerular numbers and staged sclerotic glomerular numbers were determined in the paired sections. The LC diet was specially made using $2 / 3$ of the regular calorie level with an additional $34 \%$ of protein, sodium, potassium and other nutritional contents similarly balanced to those in the control group.

\section{Telemetry Transmitter Implants and Measurements of Blood Pressure}

For accurate measurements, telemetered SBP was obtained. Radiotelemetry transmitter installation surgery was carried out at 13-14 weeks of age under methohexital anesthesia for an abdominal incision to place an aortic catheter, affixing transmitters to the abdominal wall. The accuracy of 
Table 1. Effects of Perinatal Protein and Salt Diets on Blood Pressure, Glomerular Filtration Rate and Other Functions in Offspring at 14-15 Weeks of Age

\begin{tabular}{|c|c|c|c|c|c|c|c|c|}
\hline Group & $\begin{array}{c}\text { NPNSns } \\
(\mathbf{n}=\mathbf{6})\end{array}$ & $\begin{array}{c}\text { NPNShs } \\
(\mathbf{n}=7)\end{array}$ & $\begin{array}{c}\text { NPHSns } \\
(\mathbf{n}=\mathbf{6})\end{array}$ & $\begin{array}{c}\text { NPHShs } \\
(\mathbf{n}=\mathbf{5})\end{array}$ & $\begin{array}{c}\text { LPNSns } \\
(\mathbf{n}=\mathbf{5})\end{array}$ & $\begin{array}{c}\text { LPNShs } \\
(\mathbf{n}=\mathbf{5})\end{array}$ & $\begin{array}{c}\text { LPHSns } \\
(\mathbf{n}=7)\end{array}$ & $\begin{array}{c}\text { LPHShs } \\
(\mathbf{n}=\mathbf{6})\end{array}$ \\
\hline \hline $\begin{array}{c}\text { Baseline } \\
\text { SBP (mm Hg) }\end{array}$ & $131 \pm 2$ & $137 \pm 2 *$ & $137 \pm 0.2 *$ & $134 \pm 3$ & $138 \pm 1 * *$ & $138 \pm 0.6 * *$ & $135 \pm 2$ \\
\hline $\begin{array}{c}\text { RR-SBP } \\
(\mathrm{mm} \text { Hg) }\end{array}$ & $-3.3 \pm 2$ & $-5.8 \pm 1 *$ & $-0.8 \pm 0.9$ & $-2.9 \pm 1$ & $-7.0 \pm 3 *$ & $-1.1 \pm 1$ & $-5.1 \pm 2 *$ & $-2.6 \pm 0.9$ \\
\hline $\begin{array}{c}\text { Baseline GFR } \\
(\mathrm{m} 1 / \mathrm{min} / \mathrm{g} \text { Kid) }\end{array}$ & $3.51 \pm 0.8$ & $3.49 \pm 0.7$ & $3.6 \pm 1.0$ & $4.06 \pm 0.6$ & $3.41 \pm 0.9$ & $3.66 \pm 0.4$ & $3.51 \pm 0.8$ & $4.02 \pm 0.5$ \\
\hline $\begin{array}{c}\text { RR-GFR } \\
(\mathrm{ml} / \mathrm{min} / \mathrm{g} \mathrm{Kid})\end{array}$ & $0.935 \pm 0.3$ & $0.927 \pm 0.2$ & $0.537 \pm 0.2$ & $-0.064 \pm 0.1 * *, \alpha$ & $-0.229 \pm 0.1 \dagger, \beta$ & $0.057 \pm 0.2 *$ & $-0.515 \pm 0.2 \ddagger, \gamma$ & $-0.404 \pm 0.3 \dagger$, \\
\hline RR-FI (g) & $1.05 \pm 0.6$ & $0.43 \pm 0.4$ & $1.23 \pm 0.4$ & $0.74 \pm 0.9$ & $0.63 \pm 0.4$ & $0.48 \pm 0.4$ & $0.09 \pm 0.4$ & $0.22 \pm 0.5$ \\
\hline RR-UV (ml) & $2.75 \pm 0.5$ & $2.99 \pm 1.0$ & $2.41 \pm 0.8$ & $4.12 \pm 2.5$ & $1.59 \pm 0.5$ & $0.5 \pm 1.0 \dagger$ & $2.34 \pm 0.5$ & $-1.57 \pm 1.3 \dagger+\ddagger$ \\
\hline
\end{tabular}

$\mathrm{n}=$ rat number; $\mathrm{SBP}=$ systolic blood pressure; RR-SBP $=$ renal reserve SBP; GFR $=$ Glomerular filtration rate $(\mathrm{ml} / \mathrm{min} / \mathrm{g}$ kidney); RR-GFR $=$ renal reserve GFR; RR-FI = renal reserve Food Intake; RR-UV = renal reserve Urine Volume.

Basal SBP comparison: $* \mathrm{P}<0.05 ; * * \mathrm{P}<0.01 ; * * * \mathrm{P}<0.005$ as compared to NPNSns; RR-SBP as compared to the basal SBP: *P $<0.02$; RR-GFR: NPNS vs. all LP groups: *P $<0.05$; $* * \mathrm{P}<0.025 ; \dagger \mathrm{P}<0.01 ; \sharp \mathrm{P}=0.001$ and NPHSns versus the rest 5 group comparisons: $\alpha \mathrm{P}<0.025$; $\beta \mathrm{P}<0.01 ; \gamma \mathrm{P}<0.001$ (Fig. 3b); RR-FI comparison: not significant changes in all groups; RR-UV: $\uparrow \mathrm{P}<0.05$ in both NPNSns vs. LPHSns; LPHShs vs. NPHSns, NPHShs comparisons; LPNSns and LPHSns; $\$ \mathrm{P}=\sim 0.01$ for LPHShs. vs. NPNSns and NPNShs.

telemetry SBP readings was verified by a standard mercury sphygmomanometer [1, 29-33]. Rats were returned to their home cage when they were fully awake and mobile with freely accessible food and acetaminophen in 5\% glucose solution for the first 24 hours post-surgery, followed by tap water. After a week of recovery, data was collected continuously every $10 \mathrm{~min}$ for 5 consecutive days using the Data Quest Program (Data Science International, St. Paul, MN) and converted into hourly average data to determine circadian SBPs and for the statistical comparison. By this time, all rats were gaining $\mathrm{BW}$ and $\mathrm{BP}$ was stabilized with synchronized cardiovascular rhythms.

\section{Glomerular Filtration Rate and Renal Reserve Study Procedures}

GFR was evaluated through inulin clearance. Following overnight acute $43 \%$-high protein (hp) diets as acute stress, RR-urine volume, RR- food intake, RR-SBP and RR-GFR were assessed. Baseline and RR values (hp-diet GFR - basal GFR) were obtained by the method used in our laboratory $[10,22-24]$ and were computed with food intake within \pm 2 g. For inulin measurements, the 24-h urine samples and small blood samples were collected baseline and the post- hp diet and the blood was replaced with an equal volume of isotonic saline via tail veins. Inulin was infused $(10 \%$ dissolved in isotonic saline, Sigma, St. Louis, MO) at $5 \mathrm{ml} / \mathrm{kg} / \mathrm{h}$ for $10 \mathrm{~min}$. Blood samples in duplicates $(60 \mu \mathrm{l})$ were collected into a heparinized microhematocrit tube for the baseline and $20 \mathrm{~min}$ post-infusion plasma inulin measurements. The method was modified from the human study [34]. Inulin concentrations in the urine and plasma were determined using a spectroflurometer.

\section{Statistical Methods}

Hourly average SBP data was analyzed by the linear least square rhythmometry to obtain circadian means and group comparisons [35]. For 3-way-interaction comparisons, data was analyzed by the methods of the analysis of variance and the SPSS statistical package available at the University of Minnesota Computing Center. Data is expressed as mean \pm SEM. A $p$ value of less than 0.05 was considered significant.

\section{RESULTS}

Pups were weighed at birth and Day-19. Litter size and BW were not altered by the diets of pregnant and lactating dams: BWs ranged 5.6-5.8 grams at birth; 40 grams at Day19 in each group. However, at 14-15 weeks, offspring in all 4 perinatal LP groups showed significantly lowered BW compared to all NP groups, regardless of the offspring's salt levels (Fig. 2a).

For the kidney weight $(\mathrm{KW}) / \mathrm{BW}$ comparisons shown in Fig. (2b), the offspring's hs-diet was a determining factor that resulted in a higher $\mathrm{KW} / \mathrm{BW}$ ratio than that of the nsdiet, regardless of maternal protein and salt levels (-ns versus -hs, all $\mathrm{P}<0.005$ ).

In Table 1, Baseline SBP and GFR, and values of postovernight acute high protein (hp) loaded RR-SBP, RR-GFR, RR-FI and RR-UV are presented as mean \pm SEM. Offspring in NPNShs, NPHSns, LPNShs and LPHShs groups had significantly increased SBPs, compared to the NPNSns group. While RR-SBPs were significantly lowered in LPns- and NPNS- groups, maternal HS and offspring's hs-diets affected RR-SBP inconsistently. Each group RR-FI was varied, but the change did not reach a significant level. RR-UV was significantly reduced in perinatal LP with the offspring's hsdiet (LPNShs and LPHShs) (Table 1). In baseline GFR comparisons, all groups were similar (Table 1, Fig. (3a), all $\mathrm{P}=$ not significant). RR-GFRs were significantly reduced in all LP groups following overnight acute hp-loads, especially among offspring on the hs-diet (NPNS versus each LP group, all $\mathrm{P}<0.05$ through $<0.001$, Table 1, Fig. 3b).

Maternal salt did not significantly affect glomerular maturation. However, immature glomeruli dominated by the 


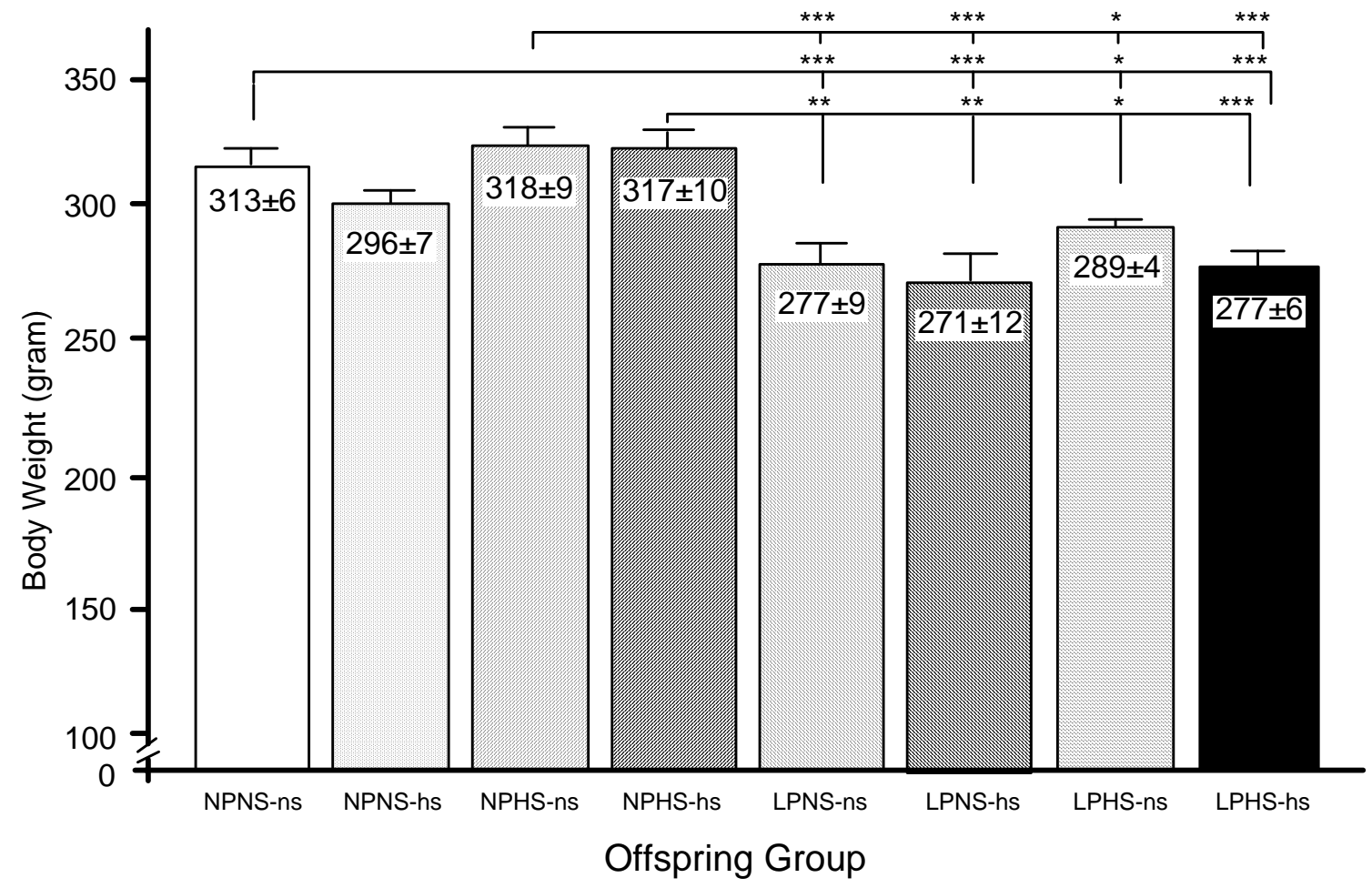

$* \mathrm{P}<0.05 ; * * \mathrm{P}<0.01 ; * * * \mathrm{P}<0.005$

Figure 2a. Offspring's Body Weight Following BP Monitoring at Day-120

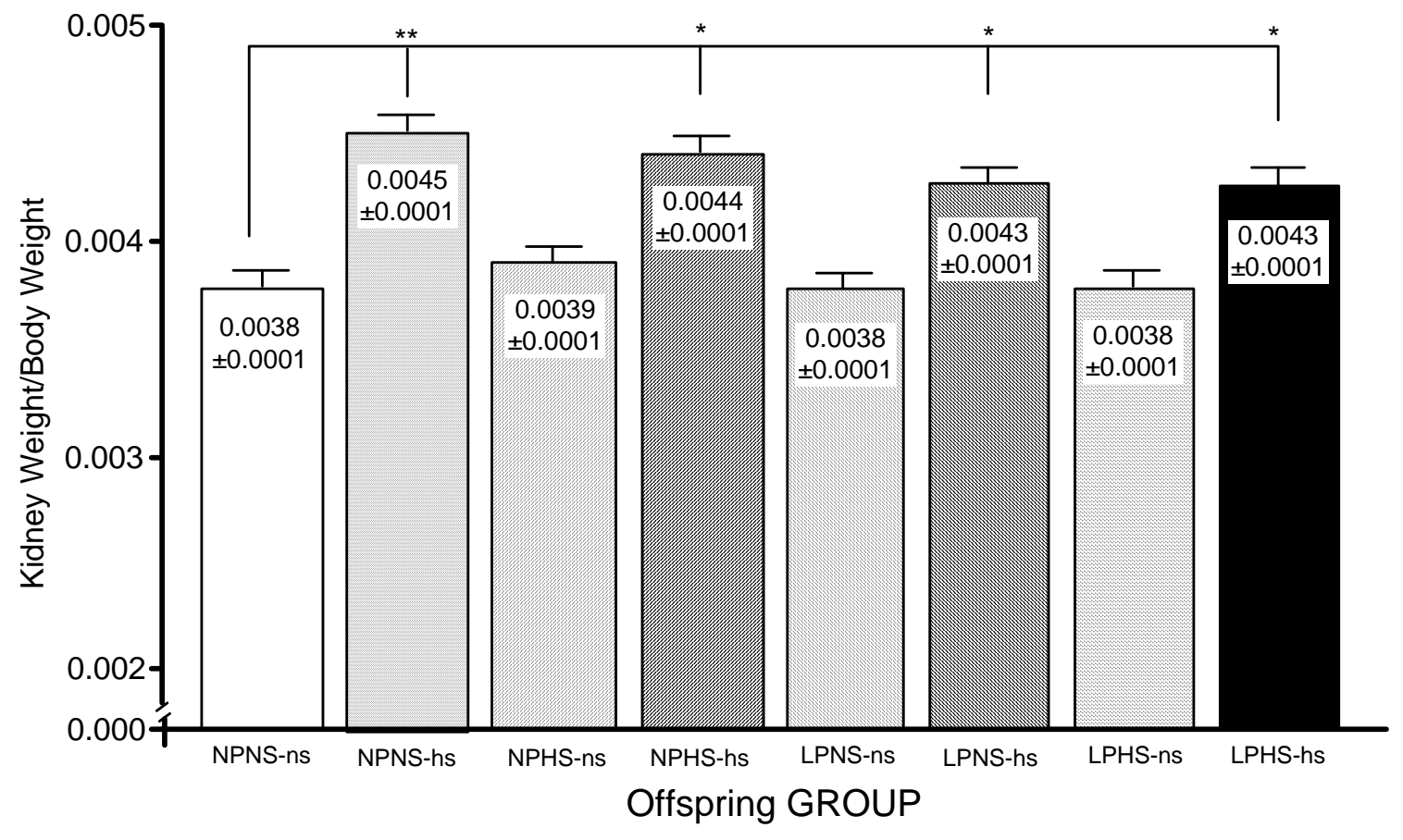

$* \mathrm{P}<0.005 ; * * \mathrm{P}<0.001$

Figure 2b. Offspring's Kidney Weight/Body Weight Ratio.

Figure 2. Effects of Maternal Protein and Salt Diets on Offspring's Body Weight and Kidney/Body Weight Ratio.

Fig. (2). Effects of Maternal Protein and Salt Diets on Offspring's Body Weight and Kidney/Body Weight Ratio. 


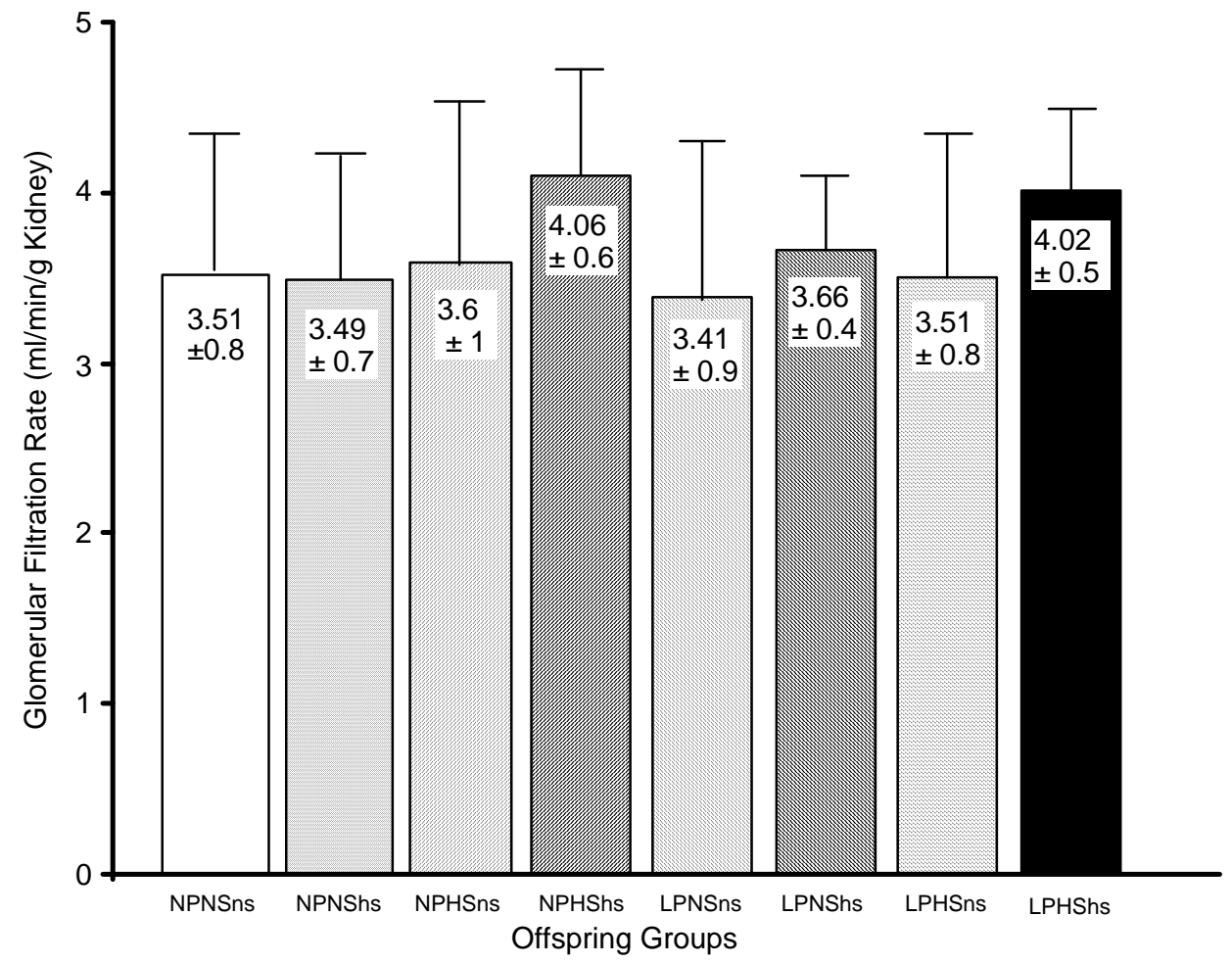

All Group Comparisons: statistically not significant

Figure 3a. Baseline Offspring’s Glomerular Filtration Rate Comparisons.

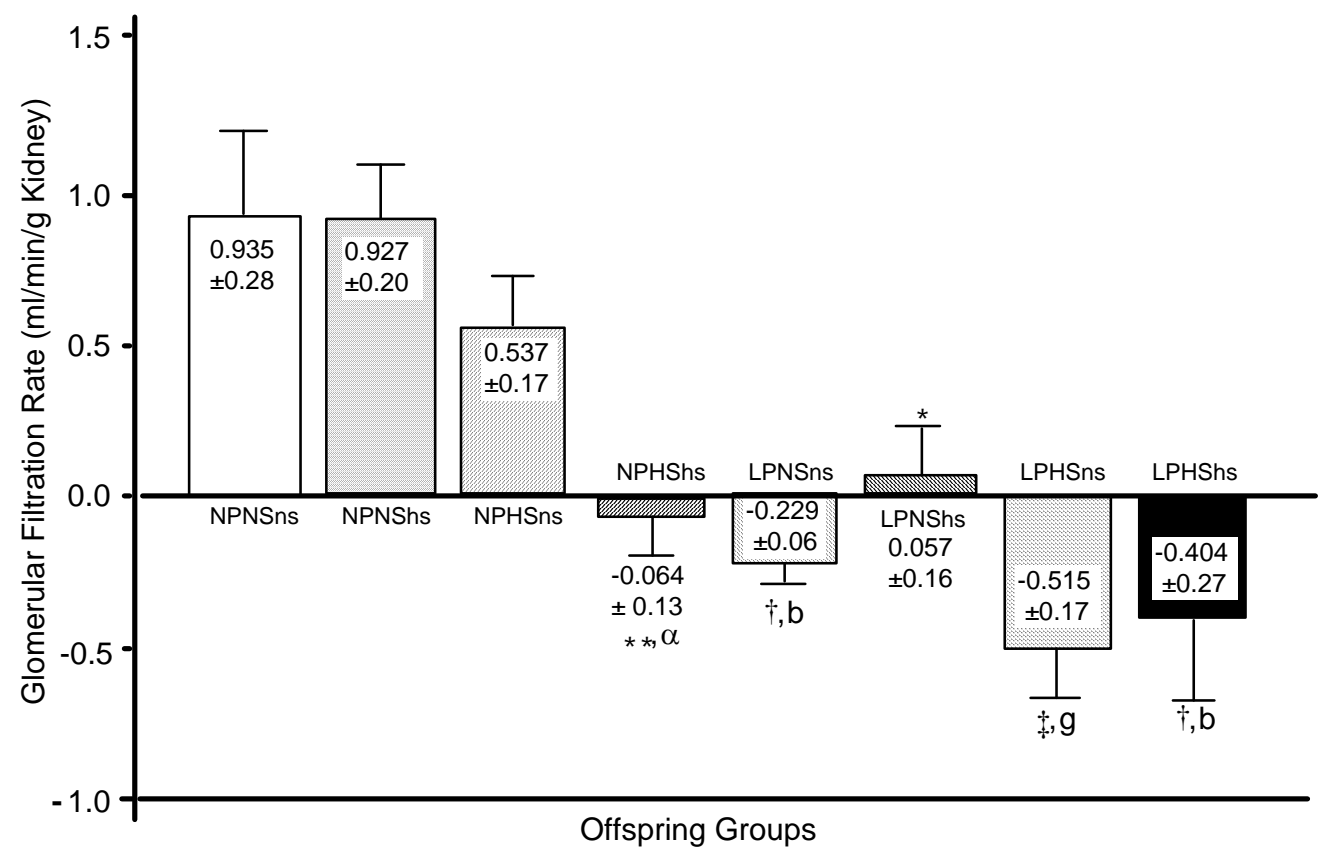

${ }^{*} \mathrm{P}<0.05 ;{ }^{*} \mathrm{P}<0.025 ; \dagger \mathrm{P}<0.01 ; \ddagger \mathrm{P}<0.001$ for NPNSns vs. all other groups and NPNShs vs. all other groups; $\alpha \mathrm{P}<0.025 ; \beta \mathrm{P}<0.01 ; \gamma \mathrm{P}<0.001$ for NPHSns vs. the rest 5 group comparisons

Figure 3b. Offspring's Glomerular Filtration Rate Changes in 8 Study Groups Following the Overnight High Protein Diet.

Fig. (3). Effects of Perinatal Protein and Salt Diets on Offspring's Glomerular Filtration Rate at 15 Weeks of Age After a High or Normal Salt Diet. 
Table 2. Effects of Perinatal Protein-Salt Diets on Progeny Glomerular Maturation at Birth and Day-7 and Proteinuria at Day-56 on a Normal Diet

\begin{tabular}{|c|c|c|c|c|c|c|c|c|c|c|}
\hline Group & \multicolumn{4}{|c|}{ Day-0 (GM Stage) } & \multicolumn{4}{c|}{ Day-7 (GM Stage) } & \multicolumn{2}{c|}{ Day-56 (Proteinuria) } \\
\hline & $\mathbf{n}$ & $\mathbf{0}$ & I & II & $\mathbf{n}$ & $\mathbf{0 - I}$ & II & III & n & (mg/24 hrs) \\
\hline \hline NPNS & 5 & $24 \pm 9$ & $67 \pm 1$ & $9 \pm 1$ & 6 & 0 & $18 \pm 1$ & $83 \pm 1$ & 6 & $5.1 \pm 1.2$ \\
\hline NPHS & 5 & $21 \pm 4$ & $64 \pm 2$ & $13 \pm 1$ & 5 & 0 & $13 \pm 4$ & $82 \pm 8$ & 6 & $5.7 \pm 1.7 \dagger$ \\
\hline LPNS & 7 & $32 \pm 8^{*}$ & $58 \pm 2 *$ & $10 \pm 2$ & 5 & 0 & $27 \pm 3 *$ & $73 \pm 3 *$ & 5 & $3.2 \pm 1.2 \ddagger$ \\
\hline LPHS & 5 & $33 \pm 9 *$ & $59 \pm 1^{*}$ & $8 \pm 2$ & 6 & 0 & $26 \pm 2 *$ & $73 \pm 2 *$ & 7 & $4.0 \pm 1.0 \ddagger$ \\
\hline
\end{tabular}

$\mathrm{n}$ : rat number; GM: glomerular maturation,

$* \mathrm{P}<0.05$ for LP vs. NP groups in GM comparisons at birth (Day-0) and Day-7;

$\dagger \mathrm{P}<0.05$ for NPNS vs. NPHS; $₫ \mathrm{P}<0.01$ for LP vs. NP group proteinuria comparisons.

low protein diet in both perinatal LPNS and LPHS groups, relative to NP groups at birth (day-0), with lower matured glomeruli numbers, all $\mathrm{P}<0.05$. This difference was persistent during the postnatal stage at day-7, with similar patterns shown as at day- 0 , all $\mathrm{P}<0.05$. At day-56, the NPHS group had the highest protein excretion: NPNS versus NPHS, $\mathrm{P}<0.05$, while perinatal LP diets lowered proteinuria significantly: NPNS versus LPNS and LPHS, both $\mathrm{P}<0.01$ (Table 2).

The calorie restriction study included only two groups with normal ( $15 \mathrm{~g} /$ day $)$ and low calorie $(10 \mathrm{~g} /$ day $)$ diets for 4 weeks. In the calorie restriction, reduced sclerotic glomerular numbers were observed as compared with the normal calorie group $(11.19 \pm 1.2$ versus $15.68 \pm 1.5, \mathrm{P}<0.001)$, though glomerular numbers were similar in both groups (111 vs. 113 per graded kidney cross section).

Table 3 represents the 3-way interaction comparisons. The combined perinatal protein, salt and offspring's diets together significantly affected offspring's SBP $(\mathrm{P}<0.014)$, while perinatal protein alone significantly affected offspring's SBP, GFR and BW ( $\mathrm{P}=0.001,0.0001$ and 0.0001 , respectively). Perinatal salt also significantly affected SBP $(\mathrm{P}=0.005)$ and RR-GFR $(\mathrm{P}=0.0001)$, and had a borderline

Table 3. Effects of Perinatal Protein and Salt Diets on Blood Pressure, Body/Kidney Weight and the Glomerular Filtration Rate in Offspring at 14-15 Weeks of Age

\begin{tabular}{|l|c|c|c|}
\hline Analysis of Variance & $\begin{array}{c}\text { BW } \\
\boldsymbol{p} \text {-value }\end{array}$ & $\begin{array}{c}\text { RR-GFR } \\
\boldsymbol{p} \text {-value }\end{array}$ & $\begin{array}{c}\text { SBP } \\
\boldsymbol{p} \text {-value }\end{array}$ \\
\hline \hline Protein--A & 0.0001 & 0.0001 & 0.001 \\
\hline Salt-- B & 0.076 & 0.0001 & 0.005 \\
\hline Pup-C & 0.363 & 0.553 & 0.567 \\
\hline A x B & 0.631 & 0.714 & 0.745 \\
\hline A x C & 0.943 & 0.013 & 0.047 \\
\hline B x C & 0.823 & 0.057 & 0.068 \\
\hline A x B x C & 0.554 & 0.950 & 0.014 \\
\hline GFR & & 0.029 & \\
\hline BW/KW & 0.755 & \multicolumn{2}{|l}{} \\
\hline
\end{tabular}

SBP: systolic blood pressure (mm Hg); BW: body weight (gram); KW: kidney weight (gram) RR-GFR: Glomerular filtration rate changes $(\mathrm{ml} / \mathrm{min} / \mathrm{g}$ Kidney) following the overnight hp-diet. A: maternal protein diet effect on offspring; B: maternal salt diet effect on offspring; C: pups' diet effect. effect on BW $(\mathrm{P}=0.076)$. Perinatal proteins and pups' salt diets together significantly affected SBP $(\mathrm{P}=0.047)$ and RRGFR ( $\mathrm{P}=0.013)$, but not $\mathrm{BW}(\mathrm{P}=$ not significant). The combination of perinatal and offspring's salt diets had a borderline effect on SBP $(P=0.068)$ and RR-GFR $(P=0.057)$.

\section{DISCUSSION}

In the current study, the main findings are that a maternal LP-diet delayed offspring's nephrogenesis and increased BP, particularly with the hs-progeny diet. Perinatal LPHS diets significantly affected offspring's BW, SBP, renal injuries and kidney functions; thus, renal reserve was seriously reduced.

Hypertension is present in more than $80 \%$ of patients with chronic kidney disease (CKD), contributing to the loss of renal autoregulatory efficiency, which may lead to glomerular stress, renal injury and progression to end-stage renal disease. This also results in an increased risk of cardiovascular events, and even the earliest stage CKD was associated with an excess risk of subsequent coronary heart disease (CHD) [36]. The interactive effects of hormones and nutrients greatly affect growth, and maternal dietary excesses and/or deficiencies can result in diverse progeny susceptibilities to systemic/local injuries that manifest in a morbidity status later in life [37]. Recent post-hoc analyses of the Modification of Diet in Renal Disease indicate that lower $\mathrm{BP}$, specifically $\mathrm{BP}<120 / 80 \mathrm{mmHg}$, may provide long-term kidney protection in patients with nondiabetic kidney disease. Regardless of the baseline daily proteinuria, the progression of hypertensive renal disease was prevented when a target BP of 130/80 mm Hg was maintained among diabetic patients [38].

Environmental factors promote not only a phenotype or disease state for the individual exposed, but also some transgenerational inheritance of phenotypes and disease states without genetic mutations or alterations in DNA sequence, with the capacity to alter the epigenome [7, 8, 39]. In humans, low birth weight occurs more frequently in disadvantaged communities with disproportionately high incidences of adult hypertension, diabetes mellitus, kidney disease and CVD, showing an inverse relationship association with hypertension in adulthood and larger glomeruli [40]. Perinatal insults, which adversely affect fetal growth and low birth weight, result in increased incidence of adult hypertension and risk of CKD [41]. The combination of small size at birth and during infancy, followed by accelerated weight gain 
from age 3 to 11 years, predicts large differences in the cumulative incidence of CHD, type 2 diabetes and hypertension [9]. The increased BP and renal injury by the perinatal insults are likely multifactorial and understanding the mechanism may lead to therapies and prevention of hypertension and the development of CKD and CVD.

Maternal environments play a role in altering the growth rate, BP and subsequent pathophysiology $[1,3,42,43]$. Recent studies reported that salt intake showed a linear relationship to SBP, while extreme salt restriction caused an increased risk for all-cause mortality [44] with "J" shaped relationships in cardiovascular events $[4,45,46]$. Since hypertension is a major risk factor for cardiovascular-renal diseases, and salt sensitivity occurs with either heredity or acquired defects in renal functions [47, 48], the salt factor remains an important issue in order to clarify the causal relationship to human diseases. Since increased BP is potentially the worst risk factor in CVD, studies need to demonstrate the cardiovascular benefits of dietary salt restriction to less than 2 grams, while BP should be monitored closely in hypertensive patients with CKD, in both clinical and home BP measurements $[49,50]$.

All of the DNA and histone methyltransferases utilize Sadenosylmethionine as a methyl donor derived from choline, methionine, or methyl-tetrahydrofolate, and can have profound and permanent effects on gene expression through epigenetic marking. Nutritional status may directly influence imprint triggering selective survival of germ cells with a particular epigenetic state in physiological metabolic stress, as well as hormonal changes to modify enzymes in response to nutritional stress $[7,8]$. Thus, perinatal protein and salt variables were manipulated in our study during the developmental stages to observe the effects on an offspring's BP and $R R$ on cardio-renal functions. Perinatal diets influence an offspring's SBP (Table 1). This occurs selectively with the offspring's hs-load as shown in the NPNShs group while an ns-diet masks RR-SBP. In contrast, SBPs were significantly lowered in LP-groups with ns-diets (LPNSns and LPHSns), but not with the hs-diet. This may suggest that epigenetic effects of the perinatal protein deficiency are masked by the protective effects of normal salt but is manifested when the offspring is exposed to the provocative hs-diet (Table 1). In our previous study, shr suckled by SHR nurses on a high salt diet had higher SBP than those suckled by SHR nurses on a low-salt diet regardless of sex, the use of anesthesia or BP methodologies [3].

Maternal dietary deficiency elicits adaptations by altering morphogenetic organogenesis during pregnancy and functional maturation during suckling that reduces and/or enlarges the RR. The offspring's hs-diet further imposes a higher work-load on the kidney and reduces RR. After the fetal stage, the lactating mother transmits nutrition to the offspring via milk in combination with abnormal renal development, which induces changes in body fluids, BP and kidney functions to further acceleration and/or aggravation of offspring hypertension. After weaning, high salt intake and other environmental factors associated with the offspring's new stage of life modulate the final course of BP development through maturity. On the other hand, overnutrition, such as a HP-diet during pregnancy, also affected dams' weight gain, newborns' BW, number of offspring, and also survival in later life [51]. The HP-diet during lactation also caused an immense risk of early sudden death with more than an eight-fold increase in offspring mortality, linked to the still incompletely explored human sudden infant death syndrome [51]. These studies indicate that balanced nutrition is important to minimize the epigenetic effect in the genesis of diseases, especially during the developmental stage of young life.

Since pre-pregnancy dietary protein intake correlates better with offspring alterations than influenced by the pregnancy itself [15], protein and salt variables were manipulated during the developmental stages of pre-pregnancy, pregnancy and lactation. Pre-pregnancy diets were given over a shorter time period of 21-27 days for a marginal deficiency prior to pregnancy, rather than the 56 days required for complete deficiency to avoid severe retardation of the offspring, making comparisons difficult between the study groups. Estrous cycle positive rats started the designated diets for 21-27 days prior to mating, and cycle positive dams were mated. We used this protocol to synchronize donor and recipient rats for our embryo transfer study, which was very effective [1]. We chose a 10\% marginal LP-level to avoid pronounced BW and $\mathrm{KW}$ differences between groups and a $4 \%$ HS-level, which is half of the known value to be toxic for pregnant rats [52]. We used a normal protein diet with $23 \%$ protein, due to the additional protein requirement during pregnancy, instead of non-pregnancy $18-20 \%$ normal protein levels. Since it maintains the vicious cycle of progressive glomerular injury, the methodology is central to the issue of vascular reactivity and BP measurements. To define SBP characteristics properly, we developed the radio-telemetered ambulatory BP measurement methodology in rats and mice, recorded multiple days for reliable ambulatory BP data to obtain the circadian SBP [1,30,31].

Maternal LP diets not only lowered BW but also affected renal maturation (Fig. 2a, Tables 1-3). Acute AA load is an accepted method for an RR test [24]. Renal functions at the stimulated level with acute AA-loads induce vasodilation and increase GFR and renal blood flow [24]. If the reserve is decreased, GFR fails to increase. Studies have demonstrated that GFR averaged similarly in both Dahl S and R rats before AA infusion, but that $\mathrm{R}$ rats consistently had a significantly increased GFR in repeated trials after AA infusions (an $81 \%$ increase), while $\mathrm{S}$ rats had no significant increase in each trial, indicating a glomerular defect [53]. In prehypertensive rats, there is already some vasodilation of the afferent and efferent arterioles to bring the normal GFR levels to some intrinsic abnormality in glomerular filtration. Since arterioles are already dilated, there can be no further dilation in response to the AA-load and no further increase in GFR. In some studies, an AA infusion and a $43 \%$ high dietary protein intake showed similar effects on GFR in 8 human subjects (14\% and $13 \%$ increased GFR, respectively), compared with values obtained while the same 8 subjects followed the lowprotein diet [54]. In the present study, we used the $43 \%$ high protein diet overnight to examine the RR effects. RR-urine volumes were also significantly reduced in perinatal LP with the offspring's hs-groups (LPNShs and LPHShs). Both protein and salt can alter symmetrical and asymmetrical growth. Reduced perinatal protein decreases utero-placental blood flow and induces asymmetrical fetal growth [3]. Thus, we expected the $\mathrm{KW}$ to be smaller relative to BW. Interestingly, 
regardless of perinatal LP and/or HS, the KW/BW ratio was consistently higher in offspring with hs-diets, when compared to that of offspring on ns-diets (all $\mathrm{P}<0.005$, Fig. 2b). The RR capacity may help assess glomerular maturation and serve as a basis to assess the effects of perinatal and offspring diets on kidney functions and injury susceptibility. Since the interactive uterine-lactation effect can be expressed later with the effects of perinatal high-salt and protein deficiency, we expected normal or low GFR in the offspring in those groups. As expected, the NPNS group showed an RRGFR that was the highest among the offspring on the ns-diet, while the offspring's hs-diet (NPNShs) did not significantly affect the RR-GFR (Fig. 3b). The NPHS group with the offspring's ns-diet produced a marginal reduction of RR-GFR, but did not reach a significant level (NPNSns vs. NPHSns, P = not significant). Strikingly, maternal LP markedly reduced RR-GFR regardless of maternal high/low salt or the offspring's salt intake levels. Thus, the perinatal protein was the determinant factor for RR-GFR. GFR, roughly proportional to the renal blood flow, largely determines the filtered loads. Therefore, when maternal RR-GFR is reduced, the hypoxic kidney resulted in increased systemic/organ stress, which may result in fetal hypoxemia, leading to adaptive responses. This suggests that the perinatal diet may "program" offspring susceptibility, which may lead to injury later in life. An offspring's hs-diet can induce a defective GFR after the maternal HS environment as shown in the NPHShs group (Fig. 3b).

In some studies, a GFR of male Sprague-Dawley rats was increased with a maternal 8\% LP diet and mean MAP was significantly lower (120 vs. $128 \mathrm{~mm} \mathrm{Hg}$ ), while offspring's $8 \%$ high salt diet did not much affect BP, although the protein restriction induced a severe nephron deficit and low glomerular volume [55]. Both the BP and GFR results were contradicted by our current results. In that study, kidney morphologic and BP measurements were done at day-135 (over 19 weeks) of offspring's age. In our study, at birth and day-7, immature glomeruli dominated in both perinatal LPNS and LPHS groups as compared with NP groups (all P $<0.05$ ), while sclerotic glomeruli numbers were significantly reduced in the restricted calorie diet. Protein excretion at Day-56 was the highest in the NPHS group and lower in both LP groups, despite a tendency of increased SBP in the LP groups as compared with the NPNS group (Tables $\mathbf{1}$ and 2). We monitored offspring's SBP at 14-15 weeks of age but omitted the glomerular maturation comparison at the end of the study at 15 weeks due to the potential distortion of the kidney status with RR tests and other stress. However, the SBP and RR-GFR results indicated that the early age immaturity continuously affected kidney function later in life. GFR was reduced significantly in male Wistar rat offspring with the $8 \%$ prenatal LP, while the LP offspring was not hypertensive during young adulthood. However, their BP was hyperresponsive to restraint stress and to salt, and GFR was more sensitive to hypertensive insults. Thus, the authors proposed that the prenatal malnutrition may predispose a hypertensive prone trait to developing hypertensive problems later in life [56]. Our result also showed a kidney deficit in the perinatal LP, but SBP was generally increased and GFR was also impaired in maternal LP- and HS- diets, especially when offspring was on an hp-diet (Table 1, Fig. 3b). Our result did not differ in glomeruli numbers measured at 4 weeks of offspring's age, although the immature and sclerotic glomeruli numbers were significantly increased in perinatal LP groups, in contrast to other study reports with reduced glomerular numbers in LP or HS maternal diets [19, 57, 58].

In understanding how metabolic programming of renal mechanisms contributes to the onset of hypertension in adulthood, authors suggested that prenatal LP offspring's renal $\mathrm{Na}^{+}$retention with a significant reduction in the whole kidney pump activity contributes to the development of hypertension $[58,59]$. Maternal protein restriction $(8 \%)$ during lactation results in alterations in GFR, renal $\mathrm{Na}^{+}$handling and in components of the angiotensin (AT) II -linked regulatory pathway of renal $\mathrm{Na}^{+}$reabsorption, suggesting the loss of the $\mathrm{Na}^{+} / \mathrm{K}^{+}$ATPase alpha-1 subunit from the inner medulla altered renal $\mathrm{Na}^{+}$handling is also programmed prenatally [59]. Both excessively high (4\%) and excessively low $(0.07 \%)$ sodium intakes during pregnancy modify protein expression in offspring kidneys and reduce the final number of glomeruli, predisposing offspring to a risk of hypertension [60]. Increased renal AT II receptor expression may arise as a result of the direct effect of protein restriction or in response to the reported decrease in renal tissue AT II concentration [61]. The authors concluded that BP may be elevated in LP rats in order to maintain GFR against a decrease in the number of nephrons with a greater decrease in GFR when challenged with AT II. This may suggest that the intact kidney may be the key to protect the overall physiological body mechanism.

The baseline SBP value of the NPNSns group $(131 \pm 2$ $\mathrm{mm} \mathrm{Hg}$ ) in our study was somewhat higher than expected. Some studies suggested that different diet manipulations in the rat pregnancy elicit different programming effects on the developing cardiovascular system [38]. LP-diets of differing composition used in different laboratories have yielded inconsistent data on the relationship between maternal protein intake and an offspring's BP, which may be the critical difference between the two LP-diet protocols that resulted in the programming of hypertension in the rat [62]. The balance of protein and other nutrients may be a critical determinant of the long-term health effects of maternal under-nutrition. As such, the diet in our current study was specially made with correct ingredients and balanced nutrients, such that telemetered SBPs would not result in artifacts.

In summary, we demonstrated that LP-HS perinatal diets resulted in an offspring's susceptibility to renal injuries and increased SBP, manifested at 15 weeks of young adult age, especially in an hs-progeny diet and the reduced renal reserve on a perinatal LP-diet. The results indicate that a maternal diet with adequate protein and "normal" salt intake is important in the normal development of offspring, which leads to the development of healthy kidneys. In addition, both LP and HP diets are independently capable of fetal/neonatal programming involving complex pathways with long lasting effects on offspring. Since low GFR is a risk indicator for cardiovascular and stroke incidences with increased BP, appropriate dietary implementations during pregnancy should be part of the strategy to reduce risks and help prevent cardiovascular and stroke incidents. Understanding the mechanism in raising BP and renal injury by perinatal insults may lead to the prevention of hypertension, CKD and CVD. As shown in the current study, perinatal LP and HS diets significantly affect the offspring's nephrogene- 
sis, SBP, renal injuries and kidney functions later in the offspring's life, especially magnifying maternal salt effects with reintroduced offspring's hs-diets that suggest the epigenetic effect. It would be interesting in examining effects of the maternal diet influenced on the 2 nd and 3rd generation offspring.

\section{CONCLUSION}

All group pups were born with similar BW. However, at 14-15 weeks, offspring in all 4 perinatal LP groups showed significantly lowered BW compared to all NP groups, regardless of the offspring's salt levels. The most interesting result is the glomerular maturation staging, which a maternal LP-diet affects by delaying offspring's nephrogenesis and increasing BP, especially with a hs-progeny diet. Perinatal LPHS diets significantly affected offspring's BW, SBP, renal injuries and kidney functions; thus, renal reserve was seriously reduced, especially when an offspring is on the hs-diet. The results may provide important information in understanding potential human perinatal nutritional requirements for healthy fetal growth, which will be beneficial to the public health.

\section{CONFLICT OF INTEREST}

None of authors in this report has conflict of interest.

\section{ACKNOWLEDGEMENT}

The authors thank Mr. John S. Lee for his critical review, comment and editorial assistance.

SOURCES OF FUNDING: This work was supported by the National Institutes of Health, Grant RO1 HL-35643 and Grant by the Minnesota Medical Foundation, Minneapolis, Minnesota.

This work is dedicated to Dr. Silvia H. Azar who worked hard toward the results but unfortunately she passed before this publication.

\section{STUDY GROUP ABBREVIATIONS}

NPNShs, NPHSns, NPHSns, NPHShs, LPNSns, LPNShs, LPHSns, LPHShs:

Maternal diets (capital letters):

NPNS $=$ normal protein/normal salt
NPHS $=$ normal protein $/$ high salt
LPNS $=$ low protein $/$ normal salt
LPHS $=$ low protein/ high salt

Offspring diets (small caps): ns: normal salt diet; hs: high salt diet

\section{REFERENCES}

[1] Lee JY, Azar SH. Wistar-Kyoto and spontaneously hypertensive rat blood pressure after embryo transfer into different wombs and cross-suckling. Exp Biol Med 2010; 235: 1-10.

[2] Gleiberman L. Blood pressure and dietary salt in human populations. Ecol Food Nutr 1973; 2: 143-56.

[3] Azar S, Kabat V, Bingham C. Environmental factor(s) during sucking exert long-term effects upon blood pressure and body weight in spontaneously hypertensive and normotensive rats. J Hypertens 1991; 9: 309-27.
[4] Cruz DN, Bagshaw SM. Heart-kidney interaction: epidemiology of cardiorenal syndromes. Int J Nephrol 2010; 2011, 351291.

[5] McCullough PA, Li S, Jurkovitz CT, et al. KEEP Investigators. Chronic kidney disease, prevalence of premature cardiovascular disease, and relationship to short-term mortality. Am Heart J 2008; 156: 277-83.

[6] Matsushita K, van der Velde M, Astor BC, et al. Collaborators (91). Asociation of estimated glomerular filtration rate and albuminuria with all-cause and cardiovascular mortality in general population cohorts: a collaborative meta-analysis. Lancet 2010; 375: 073-8.

[7] Pembrey ME. Time to take epigenetic inheritance seriously. Eur J Hum Genet 2002; 10: 669-71.

[8] Zeisel SH. Epigenetic mechanisms for nutrition determinants of later health outcomes. Am J Clin Nutr 2009; 89: 1488S-93S.

[9] Barker DJP, Eriksson JG, Forsen T, Osmond C. Fetal Origins of adult disease: strength of effects and biological basis. In J Epidemiol 2002; 31: 1235-9.

[10] Azar S, Johnson MA, Iwai J, Bruno L, Tobian L. Single-nephron dynamics in "post-salt" rats with chronic hypertension. J Lab Clin Med 1978; 91: 156-66.

[11] Meyer J, Morgan T. The effect of sodium intake on the blood pressure related to age and sex. Clin Exp Hypertens 1983; 59(A): 99118.

[12] Reisin E, Harris KY, DeBoisblanc BP, Azar S, Liuzza GF, Takur GF. The effect of low calorie unrestricted protein diet on the kidney of uninephrectomized Spontaneously Hypertensive rats. J Hypertens 1996; 14 (Suppl I): PS190 (abs).

[13] Kimura N, Toshinia H, Nakayawa Y. Population survey on cerebrovascular and cardiovascular diseases. Jpn Heart J 1972; 13: 11827.

[14] Luke RG. Can renal failure due to hypertension be prevented? Hypertension 1991; 18: 139-42.

[15] Resnick O, Morgane PJ, Hasson R, Miller M. Overt and hidden forms of chronic malnutrition in the rat and their relevance to man. Neurosci Beh Rev 1982; 6: 55-75.

[16] Pasamanick B, Lilienfeld AM. Association of maternal and fetal factors with development of mental deficiency. Abnormalities in prenatal and perinatal periods. J Am Med Assoc 1955; 159: 155-60.

[17] Rosso P, Kava R. Effects of food restriction on cardiac output and blood flow to the uterus and placenta in the pregnant rats. J Nutr $1980 ; 110: 2350-4$

[18] Conradi NG, Eins S, Wolff JR. Postnatal vascular growth in the neocortex of normal and protein depleted rats. Morphometric studies. Acta Neuropathol. (Berl) 1979; 47: 123-30.

[19] Meyer M, Lee MC, Azar S, Mastri A. Occlusion of the middle cerebral artery in normotensive and spontaneously hypertensive rats. Cerebrovasc Dis 1987; 6: 271-4.

[20] Bae PG, Bianchi G. Renal micropuncture study of normotensive and hypertensive Milan rats before and after the development of hypertension. Kidney Int 1978; 13: 452-66.

[21] Sealy JE, Blumenfield JD, Bell GM, Pecker MS, Sommers SC, Laragh J. On the renal basis for essential hypertension: nephron heterogeneity with discordant renin secretion and sodium for excretion causing a hypertensive vasoconstrict-volume relationship. J Hypertens 1988; 6: 763-73.

[22] Azar S, Johnson M, Hertel B, Tobian L. Single nephron pressures, flows and resistances in hypertensive kidneys with nephrosclerosis. Kidney Int 1977; 12: 28-40.

[23] Azar S, Johnson MA, Scheinman, Bruno L, Tobian L. Regulation of glomerular capillary pressure and filtration rate in young Kyoto hypertensive rats. Clin Sci 1979; 56: 203-9.

[24] Tobian L, Johnson MA, Hanlon S, Bartemes K. Prehypertensive Dahl $\mathrm{S}$ rats show no rise in GFR after amino acid infusion. Trans Assoc Am Phys 1988; 101: 292-6.

[25] Gunther E, Walter L. The major histocompatibility complex of the rats. Immunogenetics 2001; 53: 520-42.

[26] Grigors D, Ojeda NB, Alexander BT. Sex differences in the fetal programming of hypertension. Gend Med 2008; 5(Suppl A): S12132.

[27] Reckelhoff JF, Hennington BS, Moore AG, Blanchard EJ, Cameron J. Gender differences in the renal nitric oxide (NO) system: dissociation between expression of endothelial NO synthase and renal hemodynamic response to NO synthase inhibition. Am J Hypertens 1998; 11: 97-104. 
[28] Franco MCP, Arruda RMMP, Dantas APV, et al. Intrauterine undernutrition: expression and activity of the endothelial nitric oxide synthase in male and female adult offspring. Cardiovasc Res 2002; 56: $145-53$.

[29] Lee JY, Tobian L, Hanlon S, Hamer R, Johnson MA, Iwai J. How is the $\mathrm{NaCl}$ signal transmitted in $\mathrm{NaCl}$-induced hypertension? Hypertension 1989; 13: 668-75.

[30] Brockway BP, Mills PA, Azar SH. A new method for continuous chronic measurement and recording of blood pressure, heart rate and activity in the rat radio-telemetry. Clin Exp Hypertens A 1991; A13: 885-95.

[31] Lee JY, Azar SH. Ambulatory cardiovascular activities in LNAME-treated mice. Open Hypertens J 2011; 4: 23-32.

[32] Lee JY, Tobian, L. Aqueduct block markedly reduces mortality and hypertension in post-DOCA Dahl R rats. Hypertension 1991; 17: 1197-203.

[33] Lee MS, Lee JS, Lee JY. Prevention of erythropoietin-associated hypertension. Hypertension 2007; 50: 439-45.

[34] Claries-Appiani A,Tirelli AS, Ardissino G, Dacco V, Moretto E, Corbetta $\mathrm{C}$, et al. Hypotonic saline infusion alters the renal response to amino acids in men. Am J Physiol Renal Physiol 1999; 276: F137-42.

[35] Mojon A, Fernandes JR, Hermida RC. Chronolab. An interactive software package for chronobiologic time series analysis written for the Macintosh computer. Chronobiol Int 1992; 9: 403-12.

[36] Inscho EW. Mysteries of renal autoregulation: Lewis K Dahl Memorial Lecture. Hypertension 2009; 53(Pt 2): 299-306.

[37] Reville P, de Laharpe F, Koll-Back MH. Ross M; Enhancement of renal compensatory hypertrophy by hyperadrenocorticism and its modulation by nutritional factors. Horm Metab Res 1982; 14: 48893.

[38] Appel LJ, Wright JT, Jr Greene T, et al. for the AASK Collaborative Research Group. Intensive blood pressure control in hypertensive chronic kidney disease. N Engl J Med 2010; 363: 918-29.

[39] Skinner MK, Guerrero-Bosagna C. Environmental signals and transgenerational epigenetics. Epigenomics 2009; 1: 111-7.

[40] Luyckx VA, Brenner BM. Low birth weight, nephron number, and kidney disease. Kidney Int 2005; 97(Suppl): S68-77.

[41] Baum M. Role of the kidney in the prenatal and early postnatal programming of hypertension. Am J Physiol Renal Physiol 2010; 298: F235-47.

[42] Blizard DA, Adams N. Maternal influences on cardiovascular pathophysiology. Experientia 1992; 48: 334-45.

[43] Di Nicolantonio R, Koutsis K, Westcott KT, Wlodek ME. Relative contribution of the prenatal versus postnatal period on development of hypertension and growth rate of the spontaneously hypertensive rat. Clin Exp Pharm Physiol 2006; 33: 9-16.

[44] Taylor RS, Ashton KE, Moxham F, Hooper L, Ebrahim S. Reduced Dietary Salt for the Prevention of Cardiovascular Disease: A MetaAnalysis of Randomized Controlled Trials. Am J Hypertens 2011; 24: 843-53.

[45] Alderman MH. The Cochrane Review of Sodium and Health: Editorial. Am J Hypertens 2011; 24: 854-8.
[46] Alderman MH. Dietary sodium and cardiovascular disease: the ' $\mathrm{J}$ 'shaped relation. J Hypertens 2007; 25: 903-7.

[47] Wu X, Scholey JW, Sonnenberg H, Melo LG. Renal vascular morphology and hemodynamics in Dahl salt-sensitive rats on high saltlow potassium diet: neural and genetic influences. J Hypertens 2000; 18: 783-93.

[48] Sanders PW. Dietary salt intake, salt sensitivity and cardiovascular health. Hypertension 2009; 53: 442-5.

[49] Toto RD. Treatment of hypertension in chronic kidney disease. Semin Nephrol 2005; 25: 435-9.

[50] Levin A, Diurdiev O, Beaulieu M, Er L. Variability and risk factors for kidney disease progression and death following attainment of stage 4 CKD in a referred cohort. Am J Kidney Dis 2008; 52: 66171.

[51] Walther T. Dietrich N, Langhammer M, et al. High-protein diet in lactation leads to a sudden infant death-like syndrome in mice. PLoS ONE 2011; 6: e17443.

[52] Karr-Dullien V, Bloomquist E. The influence of prenatal salt on the development of hypertension by spontaneously hypertensive rats. Proc Soc Exp Biol Med 1979; 160: 421-5.

[53] Tobian L, Johnson MA, Hanlon S, Bartemes K. A glomerular defect in prehypertensive Dahl S rats, which limit their capacity to increase GFR. Am J Hypertens 1989; 2(7): 557-9.

[54] Badalamenti S, Ginés P, Arroyo V, et al. Effects of intravenous amino acid infusion and dietary proteins on kidney function in cirrhosis. Hepatology 1990; 11: 379-86.

[55] Hoppe CC, Evans RG, Moritz KM, Cullen-McEwan LA, Fitzgerald SM, Dowling J, Bertram JF. Combined prenatal and postnatal protein restriction influences adult kidney structure, function, and arterial pressure. Am J Physiol Regul Integr Comp Physiol 2007; 292: R462-9.

[56] Augustyniak RA, Singh K, Zeldes D, Singh M, Rossi NF. Maternal protein restriction leads to hyperresponsiveness to stress and saltsensitive hypertension in male offspring. Am J Physiol Regul Integr Comp Physiol 2010; 298: R1375-82.

[57] da Silva AA, de Noronha IL, de Oliveira IB, Malheiros DM, Heimann JC. Renin-angiotensin system function and blood pressure in adult rats after perinatal salt overload. Nutr Metab Cardiovasc Dis 2003; 13: 133-9.

[58] Luzardo R, Silva PA, Einicker-Lamas M, et al. Metabolic programming during lactation stimulates renal $\mathrm{Na}+$ transport in the adult offspring due to an early impact on local angiotensin II pathways. PLoS ONE 2011; 6: e21232.

[59] Alwasel SH, Ashton N. Prenatal programming of renal sodium handling in the rat. Clin Sci (Lond) 2009; 117: 75-84.

[60] Koleganova N, Piecha G, Ritz E, et al. Both high and low maternal salt intake in pregnancy alter kidney development in the offspring. Am J Physiol Renal Physiol 2011; 301: F344-54.

[61] Sahajpal V, Ashton, N. Renal function and angiotensin AT1 receptor expression in young rats following intrauterine exposure to a maternal low-protein diet. Clin Sci (Lond) 2003; 104: 607-14.

[62] Langley-Evans SC. Critical differences between two low protein diet protocols in the programming of hypertension in the rat. Int $\mathrm{J}$ Food Sci Nutr 2000; 51: 11-7.

Received: December 29, $2012 \quad$ Revised: February 20, 2013

(C) Lee and Azar; Licensee Bentham Open.

This is an open access article licensed under the terms of the Creative Commons Attribution Non-Commercial License (http://creativecommons.org/licenses/by-nc/3.0/) which permits unrestricted, non-commercial use, distribution and reproduction in any medium, provided the work is properly cited. 